\title{
Pricing for QoS Provisioning Across Multiple Internet Service Provider Domains
}

\author{
Soheil Saberi ${ }^{1}$, Roland P. Malhamé ${ }^{1}$, and Lorne G. Mason ${ }^{2}$ \\ ${ }^{1}$ École Polytechnique de Montréal and GERAD, Montréal Canada H3T 1J4 \\ ${ }^{2}$ McGill University, Montréal Canada H3A 2K6
}

\begin{abstract}
In this paper we introduce a pricing scheme to be employed between a group of Internet service providers (ISPs) and a customer who wishes to initiate a packet flow from a fixed origin to a fixed destination. The ISPs are transparent to the customer who relies on a third party company for both the choice of the relevant ISPs and the unit flow price negotiated. The customer pays only for that portion of the traffic, which meets a predefined maximum tolerable total delay within the ISP networks. After taking in a fixed percentage of total profit, the third party redistributes the remaining benefits to the ISPs according to a sharing mechanism, which reflects both, the QoS the ISPs declare they will meet, as well as their real performance. The pricing emerges as the result of a Stackelberg game with the third party as the leader and the ISPs as the followers 1
\end{abstract}

Keywords: Multiple Domain Internet Pricing, Game theory, Statistical Quality of Service, Stackelberg Games.

\section{Introduction}

With the advent of new Internet applications for which more quality guarantees are expected from Internet service providers, existing flat rate charging schemes have become more and more inappropriate 11. As a result, Internet pricing is currently a very active area of research. Based on the notion of effective bandwidth, a statistically founded tool for the evaluation of quality constrained bandwidth requirements for certain types of traffic in data networks [2.3], as well as different results from both cooperative and non cooperative game theory [4], various pricing approaches have been proposed.

In many schemes, along with the basic objective of pricing which is to recover the incurred costs, other goals have been considered among which, congestion control and fair allocation of resource to users [25], admission control and QoS provisioning [6], allocating the resource to users who value it most by selling the service in an online auction [7/8. As argued in 9, the profit of ISPs as major players in Internet, has been neglected in many pricing schemes; therefore in

\footnotetext{
${ }^{1}$ This research was supported by a strategic grant from NSERC, CANADA, grant:
} STPGP269449-03.

T. Chahed and B. Tuffin (Eds.): NET-COOP 2007, LNCS 4465, pp. 236-246, 2007.

(C) Springer-Verlag Berlin Heidelberg 2007 
this paper we are also interested in the interaction between ISPs and the outcome of the non-cooperative game between them. However, our model differs in a number of ways from that in 9. We have assumed the case of only one data flow that passes through designated ISPs, and the end user who initiates the process is assumed to be willing to pay only for that portion of the traffic that meets a specific delay bound. On the other hand, an ISP reward structure is defined whereby each ISP obtains a share of customer payments which depends on both its initially declared individual quality of service goals, as well as on a statistical measure of how successful this ISP is in meeting the goals in question. Furthermore, the setup here is not one of guaranteed quality of service, but rather statistical quality of service. Such a choice was made for at least two reasons: firstly, deterministic quality of service guarantees can be quite wasteful in terms of bandwidth requirements. Secondly, when involving multiple ISP domains, guaranteed qualities of service tend to require a high degree of end to end coordination, and thus the complexity and overhead communications requirements of such schemes can quickly reach unmanageable levels as network size increases. Instead here, the setup is such that the enforcement of quality of service is an affair left as entirely internal to each independent network. If a particular network complies with a high degree of success rate relative to its declared goals, it will be rewarded accordingly. If not, it will not. This way, the control scheme for quality enforcement can be left as decentralized as possible.

A third party company herein referred to as TP has been introduced as a coordinator between the end user and ISPs. In return, it receives a fixed portion of customers payments. We adopt a Stackelberg game environment, in which $T P$, is the leader, and ISPs form the group of followers.

Overprovisioning of capacities may be the solution for many network operators to deal with delay and congestion issues, but as discussed in 2, while this looks like the right choice in backbones of the network, it may not be so for its metropolitan part, and even less so in the access part of the network. This stems from the fact that overdimensioning in the latter parts requires a lot more investment and this would raise the costs as edge nodes are approached. Based on this observation, we have assumed that each ISP involved in our model has at least one congestion node along the chosen route, and the imposed delay caused by this node, dominates that of any other route link within the ISP domain. In summary, each ISP is represented by a single bottleneck node along the chosen route.

The organization of the paper is as follows. In Section 2, we describe our modelling framework. In Section 3 we specify the utility functions associated with all of the active agents. In Section 4 we present our success-rate based pricing scheme, and we establish existence of a unique Nash equilibrium for the ISP part of the Stackelberg game. This is followed by a set of examples in Section [5] while Section 6] summarizes our conclusions and plans for future work. 


\section{Model Description}

The proposed model involves three types of agents: a customer herein referred to as $C, T P$, and a collection of ISPs to be selected by $T P$. In our model, $C$ is an end user with a potentially large volume of traffic to be sent on a regular basis from a given destination $\mathrm{A}$ to a destination $\mathrm{B}$, and who initiates contacts with $T P$ for that purpose. However, $C$ specifies a maximum end to end tolerable delay for those transmitted packets for which it is willing to pay a per unit premium. We denote the maximum delay tolerated by $C$ as $T_{\max }$. An example of traffic type particularly relevant to the context here is VoIP. This is because in VoIP one can sustain the high loss probabilities that may occasionally result from the organization scheme to be proposed. Furthermore, there does already exist market regulators in the VoIP context and they can readily be identified as potential TPs in our model. Indeed the Telecom Decision CRTC 2005-28, which has been set by Canadian Radio-Television and Telecommunications Commission is a clear example of a set of regulations, upholding rather identical regulatory framework as extant traditional phone services for VoIP [10].

Division of revenues amongst telephone companies is based on mutual agreements between pairs of service providers. In the case of a large number of such providers of different hierarchical levels e.g. trunk providers and access network providers, the task of revenue sharing is currently performed by a third party company. Exchanges of balances, and information about each traversing telephone call between service providers are based on annual calculations. In the current model, TP plays an enhanced role, as compared to the case of telephone networks, in that a real-time information and revenue sharing mechanism is adopted.

$T P$ together with $C$, agree on an offered traffic versus unit flow price curve, whereby offered traffic levels increase as bandwidth unit price decreases. This curve is a form of commitment on the part of the customer that it will pay a fixed bandwidth unit price per unit time for sending a given ultimately agreed to traffic level, unless it can demonstrably establish failure by $T P$ to meet the QoS requirements at that traffic level. In the latter case, $C$ 's per unit time payment is reduced by the fraction of its total traffic inadequately transmitted. As a consequence of this arrangement, it is in $C$ 's best interest to constantly probe performance by sending traffic (useful or otherwise) at the agreed to level.

$T P$ selects a number of ISPs along the route who are willing to be solicited in offering the service to $C$. At this stage, TP gathers from the candidate ISPs the parameters which specify the rules of the game they have to play and whose outcome will be their individual share of the income.

In the practical context, we assume that packet end to end delays, and within ISP domains, can be monitored for performance verification. However, all optimization decisions are founded on specific modelling assumptions. In the current context, we have settled for a simple $\mathrm{M} / \mathrm{M} / 1$ queueing model of each network. We have assumed constant packet lengths, which without further loss of generality are taken to be of length unity, so that the probability of meeting the delay requirement can be expressed as $P\left(t \leq T_{i}\right)=1-e^{\left(\lambda-\mu_{i}\right) T_{i}}$, where $\lambda$ is the rate 
of the source, $t, \mu_{i}$ and $T_{i}$ are random delay, service rate and declared maximum transit time in network $i$, respectively.

The need to calculate success probabilities in each network, stems from the fact that we wish to reflect the customer payment mechanism on the ISPs involved in the negotiation. More specifically, the fraction of total revenue dedicated to an ISP directly depends on the probability of meeting the declared delay within its network. Moreover, as mentioned earlier, $C$ pays according to the probability that its packet reaches the destination in time; the latter probability can be derived from the probability distribution of individual network delays.

The per unit time cost for the customer will be: $\operatorname{Pr}\left(t \leq T_{\max }\right) C_{v}(\lambda) \lambda$, where $C_{v}(\lambda)$ is the unit cost versus traffic $\lambda$, dependency curve, herein referred to as the customer response curve. For convenience here, it is taken to be a decaying exponential. Indeed, anticipating a decreasing function of demand versus price is standard (see 9 for example). With all active agents and their declared parameters thus defined, we are ready to formulate the rules of a Stackelberg game whose outcome is the traffic rate submitted by $C$ to the ISPs, the corresponding premium unit flow price paid by $C$, and the revenue obtained by each of the candidate ISPs.

\section{Utility Functions and Game Framework}

\subsection{Third Party $T P$}

$T P$, is a company responsible for all negotiations with the ISPs, with the understanding that the negotiation process must remain transparent to the customer. $T P$ s unit time revenue is a fixed fraction of the total unit time payments made by $C$. The utility function of $T P$ is considered to be:

$$
T P_{U}(\lambda)=M \operatorname{Pr}\left(t \leq T_{\max }\right) C_{v}(\lambda) \lambda
$$

where $M \in[0 ; 1]$ is the fraction of total benefit reserved for $T P$. The only decision variable of $T P$ is $\lambda$, and it is chosen to maximize $T P$ 's revenue, or equally total customer payments to the ISPs, so that in a formulation of the game where ISPs cannot acquire more bandwidth, this corresponds to the social welfare optimization problem. We also assume an upper bound $\lambda_{\max }$ for the rate of data transfer.

\subsection{Service Providers}

We assume each network involved in the transaction to have a certain amount of bandwidth $\mu_{i}$, naturally available for $C$ 's traffic. Furthermore, we assume that this initial bandwidth is sufficient to insure that the maximum possible source rate $\lambda_{\max }$ can be satisfied by any of the $\mu_{i}$ 's $\left(\lambda<\mu_{i} \forall i\right)$. The ISPs have the option of increasing the amount of bandwidth they dedicate to $C$ 's traffic, via a specified cost of $c_{i}$ per unit of added bandwidth. Let $\Delta \mu_{i}$ be the 
added bandwidth with an upper bound $\Delta \mu_{i}^{\max }$, so that the actual bandwidth that network $i$ can allocate to the flow becomes: $\mu_{i}+\Delta \mu_{i}$. For each potential $\lambda$, the fraction of profit, which is not taken by $T P$, is assumed to be available in its entirety to the participating ISPs. However, for each fixed $\lambda$, ISPs are pitted against each other in a game, the rules of which will be defined in what follows. The idea is to reflect the payment mechanisms at TP's level all the way down to the ISPs. More specifically, ISP $i$ is asked to provide a (hypothetical) maximum delay $T_{i}$ that it declares itself ready to aim at meeting. This $T_{i} \in\left[0 ; T_{i}^{\max }\right]$ is very instrumental in determining ISP $i$ 's share of total income available after $T P$ 's payment, in that it is proposed that the fraction of that total allocated to ISP $i$ be given by:

$$
S_{i}=\frac{\left(1-e^{-\left(\mu_{i}+\Delta \mu_{i}-\lambda\right) T_{i}}\right)}{T_{i}^{\beta}}\left[\sum_{j=1}^{n} \frac{\left(1-e^{-\left(\mu_{j}+\Delta \mu_{j}-\lambda\right) T_{j}}\right)}{T_{j}^{\beta}}\right]^{-1},
$$

with $\beta$ as a coefficient between 0 and 1 (inclusively), and $n$ as the number of ISPs.

Also note that, the larger the declared time, the less margin is left for other providers to accommodate their own delays along the packet route. From that point of view, fairness would dictate that a large declared $T_{i}$ should correspondingly penalize the declarer (this explains the $T_{i}^{\beta}$ in the denominator in (2)). The latter penalty prevents ISPs from letting their own declared $T_{i}$ 's go to infinity in an effort to maximize their chances of success. Also, note that for an adequate choice of $\beta$ the optimal choice of declared $T_{i}$ may well become the mean delay in the network. In addition, as alluded to earlier, the ISP has the option of either buying for a given unit price extra bandwidth, or equivalently freeing, albeit at the cost of some loss of revenue per unit bandwidth, a given amount of bandwidth, thus modulating its effective service rate $\mu_{i}$. As a consequence ISP $i$, must provide two decision variables: $T_{i}$, and the extra amount of bandwidth $\Delta \mu_{i}$ it wishes to buy . Note that if we fix $\Delta \mu_{i}=0$ (no bandwidth buying allowed), it is not difficult to see that, modulo a reward shift by an appropriate constant, the game is equivalent to a zero-sum game. Using this allocation rule, we define the utility function as:

$$
I S P_{U_{i}}=(1-M) C_{v}(\lambda) \operatorname{Pr}(t \leq T) \lambda S_{i}-c_{i} \Delta \mu_{i} .
$$

where $(1-M) C_{v}(\lambda) \operatorname{Pr}(t \leq T) \lambda$ represents the revenue after payment of $T P$, and $c_{i}$ is the extra per unit bandwidth equivalent cost.

\subsection{Formulation of the Game}

While we have specified different agents utility functions, we have not thus far specified the sequence in which the game is played. Given the predominant role of $T P$ as the main organizer, we suggest that $T P$ be considered as the higher level of the hierarchy within a Stackelberg game, i.e. TP is the leader. All participating ISPs are followers, and thus, for each fixed value of customer traffic rate 
$\lambda$ decided by the leader $T P$, we shall be looking for potential Nash equilibria. We also assume a perfect information environment, whereby each player knows all extra bandwidth unit buying costs, initial networks dedicated bandwidths to $C$ 's traffic, as well the customer response curve. This strong assumption is made in order to investigate the feasibility of the ideal game. However, more relaxed versions of the game where ISP's costs per unit bandwidth are assumed unknown to TP as well as to other competing ISPs, are possible and indeed workable.

Having the position of the leader in this game, TP can predict the outcome of the non-cooperative game among the followers, for any $\lambda$. By exploiting this fact, $T P$ can specify the customer traffic level which best suits its interests.

Remark 1. Considering the expression of $I S P_{i}$ 's utility function in (3), we note that except for the share term $S_{i}$, the utility does not depend on the choice of declared maximum transit time $T_{i}$. Also for given $\left(\mu_{i}+\Delta \mu_{i}\right), T_{i}$ can be selected independently of other decision variables to maximize $S_{i}$, leaving $\Delta \mu_{i}$ as the unique decision variable of $I S P_{i}$. Furthermore, for the special case where the coefficient $\beta$ in (2) is equal to 1 , the optimum choice is $\forall i, T_{i}=0$.

Remark 2. The fact that, at least for the $\beta=1$ case, the optimal choices of declared maximum network transit times $T_{i}$ for the ISPs correspond to the highly unrealistic value of zero, justifies their characterization as declared values. This leads to a reasonable rule for sharing benefits among ISPs. Indeed, for $\beta=1$ as $T_{i}$ goes to zero, L'Hôpital's rule yields:

$$
S_{i} / S_{j}=\left(\mu_{i}+\Delta \mu_{i}-\lambda\right) /\left(\mu_{j}+\Delta \mu_{j}-\lambda\right) .
$$

(44) in fact indicates that customer payments after commission are shared among ISPs in inverse proportion to the mean packet transit time in each of the networks. Also, it can be shown that choosing a $\beta$ different from 1 is equivalent to a sharing rule where shares become proportional to $\left(\mu_{i}+\Delta \mu_{i}-\lambda\right)^{\beta}$. Thus as $\beta$ decreases, ISPs could become more reluctant to buy bandwidth.

However, more relaxed versions of the game where ISP's costs per unit bandwidth are assumed unknown to TP as well as to other competing ISPs, are possible and indeed workable. In the next section, analysis is focused on the $\beta=1$ case. For that special case, we establish the existence of Nash Equilibrium (NE) for the followers game corresponding to any admissible $\lambda$.

\section{Properties of the Followers Game for $\beta=1$}

In the telecommunication literature the throughput of the data stream (1$\left.e^{-\left(\mu_{i}+\Delta \mu_{i}-\lambda\right) T_{i}}\right) \lambda$ over mean delay $T_{i}$ is defined as the power factor. Thus for $\beta=1$ the sharing mechanism presented in (2) can be regarded as a function of each ISP's power factor $P_{i}$. More specifically:

$$
S_{i}=P_{i} / \sum_{j=1}^{n} P_{j} \quad \text { where: } P_{i}=\left(1-e^{-\left(\mu_{i}+\Delta \mu_{i}-\lambda\right) T_{i}}\right) \lambda / T_{i} .
$$


In [1] an approach based on maximization of product of power factors to allocate a fair division of flows to users, has been introduced. Indeed, this corresponds to a so-called Nash bargaining solution. Instead, in the current model, each ISP tries to maximize its power factor and has an interest in securing a high overall success rate in meeting end to end QoS constraints.

Theorem 1. Under an inequality detailed in Lemma 2 in Appendix, in the Stackelberg game defined by leader utility function (1) and followers utility functions (3) with $\beta=1$, for every admissible $\lambda$ set by the leader, the follower game admits a Nash Equilibrium.

Proof. To prove the existence of NE's we use a paraphrase of the following theorem [4]:

Theorem 2. For each player, assuming the sets of decision variables are closed, bounded and convex, and assuming that each player's utility function is continuous in all decision variables associated with all players, and strictly concave in the entries associated with its own decision variables, for every admissible combination of decisions of other players, the associated n-person nonzero-sum game admits a Nash Equilibrium in pure strategies.

The theorem above can be easily shown to hold if strict concavity is replaced by the assumption of existence of a unique maximizer for each player's utility function for arbitrary decisions made by other players. Existence of a unique maximizer is satisfied, provided utility functions can be shown to be strictly log concave in their own decision variables. See Appendix for the proof. Since, $\Delta \mu_{i} \in\left[0 ; \Delta \mu_{i}^{\max }\right]$, the set of decision variables are both convex and compact. The continuity of utility functions on the admissible decision variable set is also obvious. Therefore a Nash Equilibrium exists.

\section{Numerical Results for a Two ISP Game}

We consider the case of two competing ISPs and associate arbitrary bandwidth unit costs to them. The inputs are: $\mu_{1}=1.1, \mu_{2}=1.2$ packet $/ \mathrm{ms}, C_{v}(\lambda)=$ $e^{-(\lambda / 0.75)}, \lambda_{\max }=1$ packet $/ \mathrm{ms}, M=20 \%, \quad T_{\max }=6 \mathrm{~ms}, \Delta \mu_{1}, \Delta \mu_{2} \in[0 ; 1]$ and $c_{1}=0.075, c_{2}=0.055$. Although, a mathematical proof of the existence of

Table 1. Simulation results of two competitive ISPs for $\beta=1$ and $\beta=0.5$

\begin{tabular}{ccc}
\hline \hline & $\beta=1$ & $\beta=0.5$ \\
\hline Optimal $\lambda($ packet/ms $)$ & 0.750 & 0.708 \\
$\left(\Delta \mu_{1}, \Delta \mu_{2}\right)$ at $\mathrm{NE}($ packet $/ \mathrm{ms})$ & $(0.442,0.603)$ & $(0.275,0.360)$ \\
$\left(T_{1}, T_{2}\right)$ at $\mathrm{NE}(\mathrm{ms})$ & $(0,0)$ & $(1.88,1.47)$ \\
$\left(I S P_{U_{1}}, I S P_{U_{2}}\right)$ at NE & $(0.0588,0.0891)$ & $(0.0701,0.0961)$ \\
$T P_{U}$ at NE & 0.0536 & 0.0517 \\
$\operatorname{Pr}\left(t \leq T_{\max }\right) \%$ & 97.06 & 93.75 \\
\hline
\end{tabular}



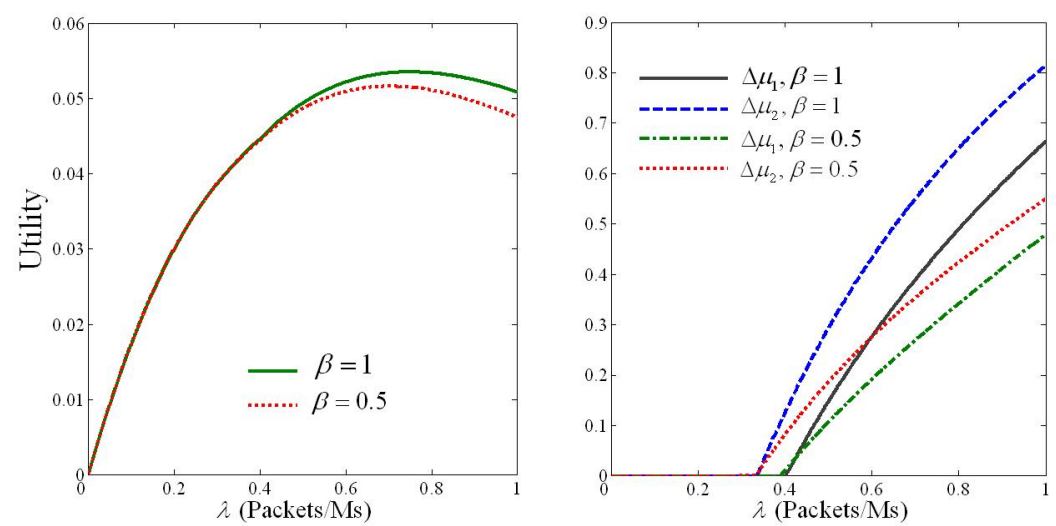

Fig. 1. Left: $T P$ 's utility versus the rate of transfer for $\beta=1$ and $\beta=0.5$, Right: $\Delta \mu_{1}$ and $\Delta \mu_{2}$ at Nash equilibria for $\beta=1$ and $\beta=0.5$

Nash equilibria for values of $\beta$ other than 1 , has not been established as yet, we numerically investigate the two cases of $\beta=1$ and $\beta=0.5$. Simulation results are shown in Table 1 and Fig 1 . From Table 1 one sees that when $\beta$ changes from 1 to 0.5 , both ISP utilities increase, but more so for the ISP with less initial bandwidth. This comes at the price of decreasing the incentives of ISPs in buying more bandwidth. This in turn lowers the QoS to the customer who has to contend with a lower probability of success.

\section{Conclusion and Future Work}

Along with the growth of VoIP and other delay sensitive Internet applications, pricing and accounting of the new services, demand new techniques and methods to better reflect each provider's performance. In this article we have proposed a scheme for rewarding Internet provider companies, which can provide low delay communications. However, no performance guarantees are given.

The global end to end performance (or equivalently the probability of meeting total delay requirements) is a result of all agents efforts to cut transit time in their own networks. This points to the importance of fair revenue sharing rules between ISPs. To deal with this issue we have investigated a class of sharing rules, parameterized by the $\beta$ variable. Setting $\beta$ at a value less than 1 , tends to reduce the financial advantage that a given ISP gets from an increase in bandwidth relative to other ISP's along the route. While this results in lower QoS, it can help offset unfair competitive advantages enjoyed by some ISP's along the route. Finding the $\beta$ that makes declared transit times equal to mean transit times, and existence and uniqueness of $\mathrm{NE}$ in the followers game for $\beta \neq 0$, are other future areas of investigation. Also in the future we will consider repeated forms of the game to account for the possibility of imperfect information, and online utility parameter estimation. Finally ISPs along the route could be divided into 
subgroups in which competition is deemed fairer, insofar as the cost of acquiring bandwidth is concerned.

\section{References}

1. DaSilva, L.A.: Pricing for QoS-enabled networks: A survey,. IEEE Communications Review 3(2) (2000)

2. Courcoubetis, C., Weber, R.: Pricing Communication Networks: Economics, Technology and Modeling. Wiley, New York (2003)

3. Kelly, F., Muallo, A., Tan, D.: Rate control for communication networks: Shadow prices, proportional fairness, and stability. J.Oper. Res. Soc. 49 (1998) 237-252

4. Basar, T., Olsder, G.J.: Dynamic Non cooperative Game. Academic Press, London and San Diego (1995)

5. Srisankar, R., Kunniyur, S.: Analysis and design of an adaptive virtual queue (AVQ) algorithm for active queue management. SIGCOMM (2001) 123-134

6. Li, T., Iraqi, Y., Boutaba, R.: Pricing and admission control for QoS enabled internet. Computer Networks 46 (2004) 87-110

7. Lazar, A., Semret, N.: Design and analysis of the progressive second price auction for network bandwidth sharing. Telecommunication Systems, Special Issue on Network Economics (2000)

8. Maillé, P., Tuffin, B.: Multi-bid auctions for bandwidth allocation in communication networks. INFOCOM (2004)

9. He, L., Walrand, J.: Pricing and revenue sharing strategies for internet service providers. IEEE/JSAC 24(5) (2006) 942

10. Canadian Radio-Television and telecommunications commission: Regulatory framework for voice communication services using Internet Protocol. (2005)

11. Mazumdar, R., Mason, L.G., Douligieris, C.: Fairness in network optimal flow control. IEEE/ACM Transactions on Networking (1990)

12. Kleinrock, L.: Queuing Systems. Volume 1. Wiley Interscience, New york (1975)

13. Avriel, M., Diewert, W.E., Schaible, S., Zang, I.: Generalized Concavity. Plenum Press, New York (1988)

\section{Appendix}

Lemma 1. The global success probability function $\operatorname{Pr}\left(t \leq T_{\max }\right)$ is strictly concave with respect to each ISP decision variable $\Delta \mu_{i}$, regardless of $\Delta \mu_{j}, j \neq i$.

Proof. The probability density function (pdf) of waiting time $t$ in a simple $\mathrm{M} / \mathrm{M} / 1$ queue is [12] $g(t, x)=x e^{-x t}$. where $x=\mu+\Delta \mu-\lambda$. The total delay $T$ that is imposed on each packet, is the sum of individual delays within each ISP's network. Thus, the pdf of $T(f(T, X))$, is the result of a convolution of all component pdf's.

$$
f(T, X)=g\left(t_{1}, x_{1}\right) * g\left(t_{2}, x_{2}\right) * \cdots * g\left(t_{n}, x_{n}\right) \text { where: } X=\left[x_{1}, x_{2}, \cdots, x_{n}\right] .
$$

Defining $F(T, X)$ as the probability distribution function (PDF) of $T$, and $X_{-i}=$ $\left[x_{1}, \ldots, x_{i-1}, x_{i+1}, \cdots, x_{n}\right]$, the pdf of total transit time when the time spent in 
ISP $i$ is excluded, can be defined as: $h_{-i}\left(T, X_{-i}\right)=g\left(t_{1}, x_{1}\right) * \cdots * g\left(t_{i-1}, x_{i-1}\right) *$ $g\left(t_{i+1}, x_{i+1}\right) * \cdots * g\left(t_{n}, x_{n}\right)>0$

The probability that the total packet delay be less than $T_{\max }$ is given by:

$F\left(T_{\max }, X\right)=\int_{0}^{T_{\max }} h_{-i}\left(t, X_{-i}\right) * g\left(t, x_{i}\right) d t=\int_{0}^{T_{\max }} \int_{0}^{t} h_{-i}\left(\tau, X_{-i}\right) g\left(t-\tau, x_{i}\right) d \tau d t$.

Using Fubini's theorem to change the order of integration in (77), we will have:

$$
F\left(T_{\max }, X\right)=\int_{0}^{T_{\max }} \int_{\tau}^{T_{\max }}\left(g\left(t-\tau, x_{i}\right) d t \quad\right) h_{-i}\left(\tau, X_{-i}\right) d \tau
$$

where $G(x, t)$ is the PDF of $g(x, t)$. Our goal is to show that $\forall X_{-i}, \quad \frac{\partial^{2} F}{\partial x_{i}^{2}}<0$.

Using Lebesgue's dominated convergence, the differentiation can be carried across the integral:

$$
\frac{\partial^{2} F}{\partial x_{i}^{2}}=\int_{0}^{T_{\max }} h_{-i}\left(\tau, X_{-i}\right) \frac{\partial^{2}}{\partial x_{i}^{2}} G\left(T_{\max }-\tau, x_{i}\right) d \tau
$$

Note that $\frac{\partial^{2}}{\partial x_{i}^{2}} G\left(T_{\max }-\tau, x_{i}\right)=-\left(T_{\max }-\tau\right)^{2} e^{-\left(x_{i}\right)\left(T_{\max }-\tau\right)}<0$ and $h_{-i}>0$; hence (9) is always negative, and as a result the global success probability is strictly concave in $x_{i}$ or equally in $\Delta \mu_{i}$.

Lemma 2. For any admissible values of decision variables $X_{-i}$, and assuming the following threshold for the total cost paid by the customer:

$$
A F\left(x_{i}, X_{-i}, T_{\max }\right)>\max \left\{c_{i}\right\} \sum_{j=1}^{n} x_{j}, \text { where: } A=(1-M) C_{v}(\lambda) \lambda,
$$

$I S P_{U_{i}}\left(x_{i}, X_{-i}\right)$ has a unique maximizer with respect to $x_{i}$.

Proof. Our goal is to show that:

$$
I S P_{U_{i}}\left(x_{i}, X_{-i}\right)=\frac{x_{i}}{\sum_{j=1}^{n} x_{j}} A F\left(x_{i}, X_{-i}, T_{\max }\right)-c_{i}\left(x_{i}-\mu_{i}+\lambda\right),
$$

always admits a unique maximizer. In Lemma 1, the strict concavity of $F\left(x_{i}, X_{-i}, T_{\max }\right)$, with respect to $x_{i}$ was established. On the other hand the function $-c_{i} \sum_{j=1}^{n} x_{j}$ is a linear function in $x_{i}$, thus the function:

$$
A F\left(x_{i}, X_{-i}, T_{\max }\right)-c_{i} \sum_{j=1}^{n} x_{j}
$$


is also strictly concave in $x_{i}$. ISP $P_{U_{i}}$ is assumed to have positive value for all ISPs and as a result, (12) is always positive. Assumption (10) ensures a positive value for (12) for all ISPs. Using Mangasarian's theorem [13, the log of (12) is a strictly concave function in $x_{i}$, and (12) will be strictly log concave. Furthermore $x_{i}\left(\sum_{j=1}^{n} x_{j}\right)^{-1}$ is also a strictly $\log$ concave function in $x_{i}$. Since log concavity is preserved under multiplication, and in view of the strictly increasing nature of the log function, the utility function in (11) has a unique maximizer in $x_{i}$. 